Resumen: La tecnología es hoy un elemento transformador que promueve nuevos modos de lenguaje y de percepción. Mirando el actual ecosistema cultural, la gestión cultural ingresó en otra dimensión. El nuevo modo de producir, ineludiblemente inscripto a un nuevo modo de comunicar, y por ende vinculado a la innovación, invita al mundo cultural a estar atento en estos nuevos comportamientos y en plantear los nuevos modos de gestionar. Instalados en el "rediseño" y considerando los desafíos del espacio cultural, se indagará en las herramientas que corresponde asumir para estos retos, tales como trazar los usos de la tecnología dentro del espacio cultural, como la coexistencia del gestor en el mundo tanto real como virtual y por el cual le permite potenciar su actuación.

Palabras claves: gestión cultural - gestor cultural - modelos culturales - innovación de gestión - redes - TIC.

[Resúmenes en inglés y portugués en la página 101]

${ }^{(*)}$ Licenciada en Humanidades (2011, Universitat Oberta de Barcelona). Se desempeñó como docente en el Curso de Gestión Cultural (2009-2011, Instituto de Formación Profesional, Severo Ochoa, Cataluña, España).

\title{
Introducción
}

En el Deviniendo virtual. La realidad en la era digital de Pierre Lévy (1998: 15-16) se preguntaba sobre cómo el mundo virtual afecta al marco colectivo, al ejercicio de la inteligencia y esencialmente a los modos de ser con los otros constituyendo un nosotros. Dentro del relato, Lévy expone "lo virtual [...] es un modo de ser fecundo y poderoso que expande el proceso de creación, que abre el futuro, que inyecta un meollo de sentido bajo la chatarra de la presencia física inmediata"; desde esta perspectiva, Lévy invita a reflexionar sobre cómo se ha extendido la era virtual en la sociedad, en las vías de comunicación y sobre todo, como continua su labor en el proceso de humanización. La sociedad vio favorecida su expresión, amplificados sus caminos de encuentro y ramificadas sus voces. Puntualmente, el hecho de poder dar una opinión de forma inmediata, conocer las diversas interpretaciones de un mismo asunto o reconocer la simpleza que se ha logrado en la interactuación con el otro, era impensable hasta hace unas décadas atrás. Todo este 
abanico de posibilidades, acarrea mayores desafíos, como el acceso, las limitaciones y las brechas originadas, entre los más significativos.

Dentro de la esfera de la gestión cultural, este salto cualitativo a nivel de comunicación, ha permitido entrar en una nueva dirección. Partiendo que la cultura defiende, por naturaleza, el contacto entre la ciudadanía y el arte, y donde procura desarrollar un dialogo que contenga la heterogeneidad de la sociedad. Desde esta mirada, el gestor cultural es un sujeto ya incuestionable, pues su función es hoy más que necesaria.

Asimismo, el poder de las TIC como medio ya ha sido más que valorado, tal como explica el termino de sociedad-red (Castells, 1999, Nonaka, 1999) donde puntualiza que "La gestión del conocimiento es más que crear bases de datos y documentos que contengan información, se refiere a la conceptualización, revisión, consolidación, creación y aseguramiento, combinando la coordinación y la recuperación del conocimiento". Desde este contexto, se profundizará sobre las nuevas tecnologías dentro del ámbito cultural.

Si se comprende la gestión cultural como acción, movimiento constante y sobre todo trabajo en red, se desprende que en esta búsqueda de generar valor agregado en el tejido social imprime un carácter emprendedor y de trabajo continuo, en el cual todos los agentes que intervienen participan de la creación de este valor. Es desde esta perspectiva el punto de inicio para reconocer los pasos dados en la era 3.0 de la gestión.

Si se comprende a la denominación 2.0, como la instancia en la cual la información fluye en múltiples dimensiones, hablar de 3.0 es utilizar toda esa información de modo personal dentro de lo profesional. Se trata del uso que se realiza sobre esa información, del cómo se utiliza, ya que se personaliza profesionalmente ese uso.

En este artículo se repasan las transformaciones dentro de la esfera cultural, gracias a la apertura del ámbito a las TIC. Mediante una contraposición entre los antiguos modelos frente a las nuevas modalidades, se observarán los cambios transitados. Ubicado en el contraste como punto de partida, se exponen las nuevas formas de administrar en el ámbito cultural, las nuevas identidades, los actores implicados y dentro del cual se despliegan los nuevos atributos y sus utilidades en la gestión cultural. La denominación 3.0 expone el paso dado, como se articula en el ámbito y lógicamente como transforma, al mayor implicado, el gestor cultural.

\section{La nueva dirección}

El modelo cultural que hasta hace poco años se encontraba vigente, se hallaba conducido desde un paradigma un tanto individualista, trabajaba desde una gestión formal, es decir, por normas rigurosas y donde la importancia jerárquica incluía la burocracia como método sistemático. No hace falta retroceder mucho años atrás para reconocer que la gestión cultural, partía inicialmente de la estrategia planteada por los marcos más institucionales, donde se proponían políticas que a mediano plazo, alcanzarían acercar a la ciudadanía propuestas acordes a los valores de cada institución. Como por ejemplo, cuando un Ministerio de Cultura, implementaba una línea de trabajo, incontables instituciones continuaban el mismo criterio. Una vez aplicadas las líneas bases, los programas llevados a cabo alcanzaban con mayor o menor éxito ciertas expectativas culturales. 
Sobre el modelo anterior, no cabe ampliar mucho, simplemente subrayar aquellos puntos ya obsoletos que se deben tener en cuenta para no volver a cometer los mismos desaciertos, donde se planifique desde las circunstancias dadas y que no queden anticuados a medio camino.

El antiguo tipo de funcionamiento cultural, dejaba a un lado las cuestiones más sensibles, como ser las manifestaciones artísticas más relegadas o menos convencionales, como por ejemplo el graffiti. Reconocía solo lo propio como lo cultural; avanzaba bajo la línea elitista marcando ciertos valores como válidos y dejando en mínimos a las cuestiones más sumergidas. Se caracterizaba por mantener una circulación lenta, es decir, que la burocracia reinaba y que buscaba la estabilidad, la homogeneidad y se hallaba sentado en el sistema de gestión analógica, en otras palabras, se trabaja desde una visión operativa.

Para mayor visualización de las transformaciones producidas, se expone un cuadro comparativo entre ambos modelos:

\begin{tabular}{|l|l|l|}
\hline PRINCIPALES DIMENSIONES & & \\
\hline & MODELO CLÁSICO & MODELO NUEVO \\
\hline RELACIÓN ESTADO & DEPENDIENTE & INDEPENDIENTE \\
\hline VALORES CENTRALES & ARTES CONVENCIONALES & APERTURA ARTÍSTICA, NUEVOS LENGUAJES \\
\hline ACTORES IMPLICADOS & INSTITUCIONES CON CARÁCTER INDIVIDUALISTA & DESCENTRALIZACIÓN, PARTICIPACIÓN LIBRE \\
\hline TIPOS DE POLÍTICAS & FORMAL Y VERTICAL & ADAPTABILIDAD Y HORIZONTAL \\
\hline DIRECCIÓN GESTIÓN & OPERATIVA & ESTRATÉGICA \\
\hline MECÁNICA DE GESTIOON & JERÁRQUICO Y BUROCRÁTICO & DIRECCIÓN PARTICIPATIVA, NUEVAS TECNOLOGÍAS \\
\hline GESTIONES CULTURALES & NO PREPARADOS ESPECÍFICAMENTE & PREPARADOS ESPECÍFICAMENTE \\
\hline MEDIOS DE COMUNICACIÓN & MEDIOS TRADICIONALES & TIC \\
\hline RESULTADOS ESPERADOS & ESTÍMULOS MORALES & NOCIONES DE VALOR \\
\hline ÁMBITO ESPACIAL & LOCAL, NACIONAL & TRANSTERRITORIAL, INTERNACIONAL \\
\hline
\end{tabular}

Figura 1. Cuadro comparativo de Modelos de Gestión. Fuente. Elaboración propia.

Como se observa, los cambios han sido esenciales y profundos. En estos últimos años se han adoptado políticas orientadas a las nociones de valor, a la satisfacción del usuario, como así a implementar una gestión que trabaja por la mejora continua, la calidad y el aprendizaje constante, alcanzando unas políticas más participativas. Asimismo, la entrada en la globalización con la suma de las TIC como conector, abrió un mundo transversal, transterritorial y de rápida circulación. La gestión cultural se explayó, se ramifico dando, por fin, con la creación de un ámbito más ajustado a sus funciones, de peso y fundamentalmente construyendo a la cultura como un bien consumible, edificando un constante ecosistema cultural. Frente a esta nueva situación, el sistema actual se extendió, se especializo y dejo al descubierto varios espacios que debe ocupar el sector y que se desarrollan más adelante. Este nuevo mundo tecnológico que despliega una fuerte imaginación colectiva, propone una eclosión de creatividad y es más, brinda un sinfín de oportunidades al ser humano. Actualmente, somos espectadores de una época muy implicada en lo cultural, donde la creciente mundialización expone al hecho cultural como valor de la sociedad vigente. Como se aprecia en el cuadro comparativo, los avances a nivel estratégicos, como así la adaptabilidad y la horizontalidad, han dado mayor potencia a la gestión procurando re- 
sultados que mejoren la coexistencia social. La evolución ha sido tan significativa, tanto para los gestores, como para la ciudadanía.

Frente a esta explosión cultural, la gestión cultural se ha visto alterada, donde la capacidad de reacción no logra alcanzar al propio movimiento. En palabras de Alfons Martinell (2003, p. 155) "la creciente mundialización ha sorprendido a unas desprovista políticas culturales que no disponían de suficiente solidez ni herramientas para afrontar estos procesos tan complejos", dejando al descubierto la dificultad, en tiempo y forma, de las existentes políticas culturales. Si bien es justo reconocer que las políticas del siglo XX han obtenido muchos adelantos e importantes transformaciones sociales, como el hecho que las políticas culturales no son feudo del Estado, sino que pertenecen al tejido social, sumado a que se ha involucrado al Estado como un elemento más dentro del aparato cultural. Por lo tanto, ahora se requiere considerar aquellos dispositivos, como por ejemplo, los despliegues de las artes combinadas, que no han podido ingresar dentro del tiempo anterior. Se entiende, aquellos procedimientos de lenta velocidad, donde la acción llega cuando ya se encuentra fuera del tiempo de la propia necesidad. Hoy se requiere ser más flexible en las gestiones y sobre todo humanizarla, para poder lograr ese dialogo que tanto se profeta. El punto de inflexión de la era actual lo manifiesta Martinell en su discurso al exponer

El aumento de participación de la ciudadanía en la cultura ha provocado la estructuración social de la comunidad en forma de organizaciones e instituciones que, desde la propia sociedad civil, sin ánimo de lucro y al margen de las estructuras del Estado, han creado un nuevo agente social denominado tercer sector de la cultura, que ha incorporado grandes iniciativas y una dimensión nueva al dialogo entre el interés general y el mercado. La emergencia y desarrollo de este tercer sector cultural es uno de los fenómenos más importantes que se están desarrollando desde el nivel local al global, generando la presencia de un nuevo actor, que hasta estos momentos no se había considerado y permitiendo un mayor desarrollo, impacto y extensión de las políticas culturales gracias a la colaboración de este sector social (Martinell, 2003, p. 157).

Es desde este enfoque, donde este nuevo sector ha generado, ha proporcionado a la sociedad, otro modo de concebir cultura.

\section{Nuevas identidades}

Moldeado un lenguaje transnacional, que circula a una rapidez hasta hace poco tiempo atrás impensable, se ha dado forma a la creación de nuevas identidades. Estos nuevos perfiles, donde se configuran con flujos de información transversal, con imaginerías fragmentadas y sobre todo, producidas con otros modos de representación que cosechan novedosas modalidades de ciudadanos. Como expone Jesús Martin-Barbero :"identidades de temporalidad menos largas más precarias, dotadas de plasticidad que les permite amalgamar ingredientes que provienen de mundos culturales muy diversos y, por lo tanto, 
atravesadas por discontinuidades" (Martin-Barbero, 2003, p. 147) accediendo a las nuevas singularidades de ciudadanía. Todo este tipo de transformaciones, cambian inevitablemente la identidad individual apuntando a la multiplicidad, a la integración y a la subjetividad que se pondera como una conquista.

La cultura sale reforzada de esta situación, permitiendo dar espacio a nuevos y distintos exponentes, conceptos y realidades. Todos estos mecanismos forman el domino cultural, permitiendo crear otros sentidos, otros comportamientos que también reproducen lo social, ahora fragmentado. En palabras de Eric Corijn

No podemos seguir hablando de la cultura como si fuera un solo objeto, un dominio en el que cada pieza encaja con otra. Tenemos que reconocer la naturaleza fragmentada y contradictoria de lo cultural [...] Hemos aprendido a vivir juntos en comunidad basados en la identidad. Ahora tendremos que aprender a vivir juntos en la diferencia (Corijn, 2003, pp. 85-87).

prevaleciendo lo distinto sobre todas las cosas. Ahora la libertad cultural empuja a construir el mercado, a negociar e interactuar desde nuevos modos de relación tanto a partir de lo político, lo económico, lo social y, por supuesto, cultural.

La creación, tanto artística como de la propia gestión cultural, se introduce en otros códigos donde prevalece un carácter atrevido y agitador, ya que propone nuevas sinergias y despierta a las veteranas contradicciones, transportando aún más a la cultura para el uso cotidiano.

\section{El artista contemporáneo}

Con la entrada de las TIC en la cultura, el artista que también hace uso de ellas, da paso a unas nuevas habilidades. Décadas atrás, generalmente, el artista se involucraba en mínimos, en las estrategias de venta o en la difusión de su trabajo, dejando esas tareas a sus representantes. El artista de esta época ha construido un espacio mayor dentro de sus tareas, ahora participa mucho más de su gestión.

Pero se debe reconocer su doble función y el valor de su trabajo. Como explica Luis Stolovich "La fuerza del trabajo es el principal factor de la producción cultural. [...] el factor trabajo calificado (creativo, artístico) es decisivo para la producción” (Stolovich, 2003, p. 226), en otras palabras, el talento es la base esencial para la industria. No existe industria que requiera de tanto ensayo para alcanzar su producto, siendo este, único e irrepetible. De ahí el valor extraordinario del arte, pero que como sector económico, se encuentra inmerso en la lógica económica, más allá de sus particularidades.

De cara al artista contemporáneo, actualmente ya se reconoce dentro de dicha lógica, comprendiendo que su trabajo pertenece a un medio económico como el resto de sectores. Abandonado el "por amor al arte", el artista actual trabaja y produce igual que el resto de industrias, es decir, sopesando el valor económico.

Si bien se trata de un sector con carácter informal, donde la irregularidad y las perspectivas son inciertas, el artista continúa acercando su trabajo a la esfera del trabajo profesional, 
como cualquier otro tipo de trabajo, donde se lucha por unos valores y compensaciones. Dicha lucha de reconocimientos ha introducido al artista a saber moverse, a conocer la lógica económica, desarrollando nuevas habilidades paralelas a su trabajo artístico. Hoy en día, el artista ya conoce mejor sus deberes y obligaciones. Según Montse Arbelo y Joseba Franco (2011) "El artista contemporáneo, adquiere nuevos roles que van más allá del propio acto creativo. Podemos hablar del artista-productor, artista-comisario, artista-investigador y por supuesto, del artista-gestor." De cara al gestor cultural, es esencial comprender que el artista ya está inmerso en la dinámica tanto económica como de la propia gestión.

\section{El gestor cultural actual}

Con un perfil emprendedor, con capacidades de alianza y autosostenibilidad, el actual gestor cultural procura generar valor agregado en sus emprendimientos. Dichos proyectos, ya sean de pequeño, mediano o gran formato, el gestor trabaja desde la búsqueda de nuevos modos de dialogo social, como así resolver los desafíos de la sociedad actual y la inquietud constante. El gestor cultural explota oportunidades, reconoce sinergias entre los sectores públicos y privados y estimula tanto su propia creatividad como la de aquellos que lo acompañan.

Toda esta batería de habilidades y aptitudes, que han nacido desde la necesidad, ya sean propias o del entorno, expone las particulares que se deben tener en cuenta a la hora de encarar la cultura, dejando entrever que las dificultades para llevar adelante un proyecto son múltiples y diversas y por eso, es difícil precisar un perfil ideal de gestor cultural, que ofrezca fórmulas a todas las situaciones por igual. Más allá de contar con conocimientos académicos, hoy en día se requiere que el gestor tenga nociones tanto de administración, como de contabilidad, de economía y de mercado, es decir, se requiere mayor versatilidad y menor grado de especialización. Evidentemente, es indispensable que posean conocimientos de la historia y de la cultura del territorio donde se desempeña su acción; por eso, se lo reconoce como un excelente agente conector. $Y$ esa diferencia, ese saber moverse, saber conectar, saber reajustar, es el motor de la nueva gestión cultural.

\section{La gestión 3.0}

¿A qué se podría llamar gestión 3.0? Actualmente las TIC han aumentado las posibilidades, pero también han elevados los niveles de trabajo que se deben tener presentes. Está claro que la presencia física es el primer gran punto en cualquier tipo de gestión, pero ahora, si no sabe comunicar por los nuevos canales, como ser las redes sociales, o reconocer todas la modalidad del estar presente en múltiples mundos virtuales, deja plasmado la dificultad de no saber alcanzar al público. Este efecto multiplicador de las redes genera adentrarse ante un verdadero cambio de época.

En palabras de Joël de Rosnay, la dirección es de "renaturalizar", donde se busca humanizar las nuevas tecnologías. Por eso, es esencial conocer el valor que soportan, las propiedades que custodian y que emergen en este nuevo ecosistema. Continuando al autor, "El tiempo 
amplio es el del capital tiempo, el que pone en paralelo el tiempo y la información [...] Abrazar información no es adquirir saberes. [...] Lo importante es integrar esas informaciones en saberes y esos saberes en culturas " (de Rosnay, 1995, p. 218). De esta manera, Rosnay explica como valorar y esencialmente como trabajar esa información, integrándola para la edificación, en este caso, de la gestión cultural. La dirección está indicada, el trabajo es construir día a día en ambos mundos, el real y el virtual.

La gestión ya se encuentra dentro de una nueva dinámica, donde sin siquiera haber tenido la posibilidad de encontrarse o cruzado con el interesado, en menos de unos minutos, el gestor ya puede conocer los intereses, gustos o curiosidades del interlocutor con el cual se desea realizar una próxima gestión. Este simple hecho proporciona una cuantiosa información que ya se utiliza para la reunión. Entonces ¿en qué deriva? En que los encuentros laborales suelen encararse desde otro enfoque, aprovechando la nueva información y proporcionando datos relevantes para el interlocutor, es más, este tipo de información ayudar a aproximar a las partes.

La mediación tecnológica abre, expone y permite el acceso a otro canal de comunicación. Tal como explica Martín-Barbero "la tecnología remite hoy no tanto y solo a nuevos aparatos sino a nuevos modos de percepción y de lenguaje, a nuevas sensibilidades y escrituras" (Martín-Barbero, 2003, p. 138). Hoy se puede comprender, que la gestión se encuentra más orientada horizontalmente, donde se permite el acceso, ya sea a la información o a las personas, de una manera más habitual para todos los profesionales del sector. Desde esta nueva posición, sumada a la batería de herramientas, se alcanza a construir otros gestores, donde cuentan con mayores instrumentos para llevar adelante los proyectos. Hoy los gestores culturales son más profesionales, cuentan con más espacio dentro de la esfera cultural y son más necesarios para el propio medio.

La novedad de las TIC están más que a la vista por todos, pero donde hay que dirigir la atención es a la construcción del sujeto profesional dentro de este medio. En palabras de Philippe Quéau "los mundos virtuales introducen en nuestra experiencia nuevos tipos de espacios y nuevas formas de vivir en esos espacios" (Quéau, 1995, pp. 95-97), es decir, que estos nuevos hábitat del ser humano, construyen otras forma de presencia, $\mathrm{y}$ tal como sentencia Quéau "nos hacen experimentar nuevas formas de ser, nuevos medios de hacernos mutuamente presentes” (Quéau, 1995, pp. 95-97). Esta mutación innovadora, empuja al gestor a construir su perfil virtual, a manejarse con otros códigos y por ende, a aplicar estos nuevos instrumentos de modo profesional. Este límite estrecho, entre lo personal y laboral, que se juega en el ciberespacio, exterioriza, con mayor énfasis sobre la esfera cultural, es decir, sus intereses concretos por la música, las artes escénicas, las audiovisuales, etc. De esta manera, se conoce aún más, al interlocutor y se convierte en una inevitable herramienta para las futuras gestiones. Este quiebre de barreras y de permeabilidades, juegan ahora un papel más que fundamental, acortan los caminos y permiten que la proximidad cibernética pueda dar otros frutos, de cara a la propia gestión, mayor contacto o más fluido, e incluso originar una afinidad.

En esta época donde se trabaja teniendo en cuenta las necesidades cambiantes y exigentes, donde se requiere de mayores colaboraciones, como así soluciones de gestión más creativas y rentables, las nuevas formas de comunicaciones a nivel de relaciones están en alza. Ahora se buscan voluntades aplicadas, simbiosis rentables y sobre todo, hacer más 
humana la gestión. Y parece increíble, pero estas nuevas dinámicas de conexión, le ofrecen a la gestión, construir una gestión cultural más humanizada. Mientras que antiguamente las relaciones entraban en el mercado de la burocracia ahora es cada uno quien hace su marketing y puede venderse como le interesa o crea oportuno.

De la misma manera, esta fórmula debe aplicarse a todos los agentes dentro del sector. Los artistas y creadores tienen aplicaciones de gestores, ya saben ofrecer y venderse, conocen los acuerdos y las estrategias, con lo cual sube el listón y colocan al gestor frente a un mayor reto. Entonces, ¿que debe ofrecer como valor agregado un gestor cultural?

Actualmente se proyecta en la esfera del gestor: ser todavía más profesional, poseer mejores herramientas y buscar cada vez más opciones para construir ese dialogo social. Tal como describe Martín-Barbero, se pretende que sea un trabajador multifacético "dotado de destrezas varias provenientes de diversos campos del saber que le permiten adaptarse a la movilidad que hoy transforma y desfigura velozmente el mapa de las funciones requeridas por los modelos de producción, de gestión y de comunicación" (Martín-Barbero 2003 , p. 139). Se pretende que el gestor conozca mejor la propia ingeniería del medio cultural, donde alcance a racionalizar tanto el dinero como los esfuerzos, sumados a lograr un importante impacto. Se apuesta por construir agentes culturales que sean inversores de desarrollo, generadores de servicios culturales consumibles, procuradores de nuevas reglas de juegos, ser aceleradores de gestiones y precursores de la innovación social, entre los puntos más relevantes.

Retomando la línea de reflexión de Lévy, se está encaminando como el propio autor manifiesta llamando el "devenir otra", ese otro devenir más próximo y comprometedor, que abre canales y que trabaja por construir ese sujeto colectivo heterogéneo que ofrece la esfera cultural.

\section{Los retos}

La relación adyacente entre la cultura y las nuevas tecnologías, sitúan a la cultura en el centro del debate, tal como explican Francesc Badia y Omar Rincón

Las nuevas tecnologías de la información y de la comunicación ofrecen una gran oportunidad para el desarrollo de la creatividad y de la diversidad cultural [...] Por eso el sector cultural tiene esta dimensión estratégica. Por eso el sector tiene una gran responsabilidad en inventar los usos de la tecnología para transformar los valores éticos y estéticos de nuestra sociedad (Badia y Rincón, 2003, p. 283).

Desde este enfoque, se comprende que la gestión cultural asume mayores desafíos a la hora de continuar desarrollando este dialogo social. La sociedad globalizada y el consumo masivo han sumado hitos sustanciales en la cultura y su gestión. Es de esencial valor que tanto la creación, como la gestión cultural indaguen todavía más en la adaptación a los 
nuevos cuadros sociales y técnicos por medio del uso de las nuevas tecnologías. Por eso la figura del gestor cultural, requiere continuar el camino de la profesionalización, dotando de contenido y enfatizando su propio espacio para poder ofrecer aún más a la cultura.

Desde esta perspectiva, el gestor cultural ocupa un eslabón fundamental, acercando, como ya se ha mencionado, los intereses ciudadanos. Como subraya Eduardo Nivón Bolán sobre el beneficio de estas relaciones virtuales expone que "El trabajo en red, por lo tanto, no está reñido con los objetivos del desarrollo, sino que es uno de sus principales aliados y tal parece que mientras más sólidos sean los lazos tendidos, mayores beneficios" (Nivón Bolán, 2003, p. 168) considerando que la construcción de redes favorece a todos los implicados. Este logro alcanzado y que da pasos más agigantados hacia la humanización, empieza a rediseñar aquel individuo simbiótico que inmerso en los tiempos de las nuevas tecnologías despliega aún más a un sujeto que vive en ambos mundos. Esta dualidad ya implícita es la que se debe potenciar de cara a la gestión.

Se puede precisar, esta apertura da más aun a aquellos que no tienen una posición de todo propicia y según Eduardo Nivón Bolán "La conformación de redes es ante todo un recurso para potenciar la actuación de los que están privados de poder" (Nivón Bolán, 2003, p. 166), es decir, esta apertura que potencia de modo horizontal provee aún más a todos por igual.

Con toda esta batería de herramientas, el nuevo gestor cultural se enfrenta a alcanzar mejores posiciones dentro del medio, optimizando los recursos, ampliar y compartir sinergias beneficiando proyectos, y por supuesto lograr crear proyectos que movilicen, tal como expresa Lluís Bonet "capaces de propiciar la interacción y la apertura hacia el otro, debería ser una de las prioridades del gestor cultural contemporáneo” (Bonet, 2011).

\section{Reflexiones finales}

Ya reconocidas las funciones y beneficios de las TIC dentro de la gestión cultural, ahora le toca zambullirse todavía más al propio gestor cultural. Dentro de este perfil multifacético, la convivencia en ambos mundos es ya una exigencia implícita.

Comprendiendo a la gestión cultural como una acción proactiva, la meta es transformar la gestión de acuerdo con las nuevas prioridades, donde se requiere continuar con la apertura y la participación. Como sentencia Martinell "Estos nuevos modelos han de intentar alcanzar una mayor participación, aportando legitimidad y democratización a la acción cultural, garantizando su diversidad y evitando el dominio de la cultura por una minoría" (Martinell, 2003, p. 159). Todo este tipo de acciones favorece el sentido común y la capacidad crítica social.

El gestor cultural, con su representación de múltiples diálogos, es en la actualidad un eslabón indispensable de la cultura. Por eso, el gestor cultural profesional empuja, con ese perfil innovador, a hacer de las nuevas tecnologías uno de sus mejores aliado para continuar desarrollando ese dialogo que representa. 


\section{Referencias Bibliográficas}

Arbelo, M., Franco, J. (2011). El artista gestor de sí mismo. (Ed.) Centro Cultural de España en Buenos Aires. Extraído el 10 Julio de 2011 de http://www.cceba.org.ar/v3/ficha. php?id=534

Badia, F, Rincón, O. (2003). Cultura y Sociedad de la información. Cooperación Cultural Euroamericana II Campus Euroamericano de Cooperación Cultural. Cartagena de Indias, Colombia, 10 al 14 de diciembre de 2001(pp. 283). (Ed.) Organización de Estados Iberoamericanos para la Educación, la Ciencia y la Cultura (OEI).

Bonet, L. (2011). El gestor cultural territorial y el formador frente al reto digital (Ed.) Bloc de Lluís Bonet i Agustí. Extraído el 10 Julio de 2011 de http://lluisbonet.blogspot. com/2011/01/el-gestor-cultural-territorial-y-el.html

Castells, M. (1999). La era de la información: economía, sociedad y cultura. Vol. 1. México: SigloVeintiuno. Extraído el 10 Julio de 2011 de http://gctercersector.wordpress. com/2008/05/28/la-gestion-del-conocimiento/

Corijn, E. (2003). Lo local en el proceso de globalización cultural. Cooperación Cultural Euroamericana II Campus Euroamericano de Cooperación Cultural. Cartagena de Indias, Colombia, 10 al 14 de diciembre de 2001. (pp. 85-87). (Ed.) Organización de Estados Iberoamericanos para la Educación, la Ciencia y la Cultura (OEI).

Lévy, P. (1998). Deviniendo virtual. La realidad en la era digital. Nueva York: Plenum Press. (pp. 15-16).

Martín-Barbero, J. (2003). Las culturas en el nuevo entorno tecnológico y el intercambio global.Cooperación Cultural Euroamericana II Campus Euroamericano de Cooperación Cultural. Cartagena de Indias, Colombia, 10 al 14 de diciembre de 2001(pp. 147). (Ed.) Organización de Estados Iberoamericanos para la Educación, la Ciencia y la Cultura (OEI).

Martinell,A.(2003).Políticas Culturales en dialogo.Cooperación Cultural Euroamericana II Campus Euroamericano de Cooperación Cultural. Cartagena de Indias, Colombia, 10 al 14 de diciembre de 2001 (pp. 155). (Ed.) Organización de Estados Iberoamericanos para la Educación, la Ciencia y la Cultura (OEI).

Nivón Bolán, E. (2003). Redes de investigación. Hacia una estrategia de integración. Cooperación Cultural Euroamericana II Campus Euroamericano de Cooperación Cultural. Cartagena de Indias, Colombia, 10 al 14 de diciembre de 2001(pp. 166). (Ed.) Organización de Estados Iberoamericanos para la Educación, la Ciencia y la Cultura (OEI).

Nonaka I. (1999). La organización creadora del conocimiento. Como las campañas japonesas crean la dinámica de la innovación. Trad. Martín Hernández Kocka. México. Oxford University Press. Extraído el 10 Julio de 2011 de http://gctercersector.wordpress.com/2008/ 05/28/la-gestion-del-conocimiento/

Quéau, P. (1995). Lo virtual. Virtudes y vértigos. Buenos Aires: Paidos. (pp 95-97).

Rosnay, J. D. (1995). Réplicas. La utopía del cibermundo. France Culture con Joël de Rosnay, Paul Virilio y Alain Finkielkraut. Versión de M. L. G. Buenos Aires: Biblio. (pp 218). 
Stolovich, L. (2003). La lógica económica del empleo cultural. Cooperación Cultural Euroamericana II Campus Euroamericano de Cooperación Cultural. Cartagena de Indias, Colombia, 10 al 14 de diciembre de 2001. (pp. 226). (Ed.) Organización de Estados Iberoamericanos para la Educación, la Ciencia y la Cultura (OEI).

Summary: Technology is now a transformative element that promotes new ways of language and perception. Looking at the current cultural ecosystem, cultural management entered into another dimension. The new way of producing, inescapably inscribed to a new way of communicating, and thus linked to innovation, invites the cultural world to be aware of these new behaviors and new ways to manage. Installed in the "redesign" and given the challenges that cultural space presents, we will explore the adecuate tools to be assumed for these challenges, such as laying out the uses of technology in the cultural space, the manager coexistence in the world both real and virtual and which allows him to enhance his performance.

Keywords: cultural management - cultural manager - cultural models - ICT - innovation management - Networking.

Resumo: A tecnologia é hoje um elemento transformador que promove novos modos de linguagem e percepção. Olhando o atual ecossistema cultural, a gestão cultural ingressou em outra direção. O novo modo de produzir, inscrito a um novo modo de comunicar, e, portanto, vinculado à inovação, invita ao mundo cultural a estar atento nestes novos comportamentos e em planear os novos modos de gestão.

Instalados na regeneração e considerando os desafios do espaço cultural, se analisarão as ferramentas que corresponde assumir para estes retos, tais como traçar os usos da tecnologia dentro do espaço cultural, como a co-existência do gestor no mundo tanto real como virtual e por o qual lhe permite potenciar sua atuação.

Palavras chave: gestão cultural - gestor cultural - modelos culturais - inovação de gestão - redes TIC. 